\section{COVID-19 and older adults: experience in Hong Kong}

\author{
Tung Wai Auyeung ${ }^{1}$, MD, FHKAM (Medicine), Felix HW Chan ${ }^{2}$, FRCP (Edin, Glasg, \\ Ire), FHKAM (Medicine), TY Chan ${ }^{3}$, MB BS, FHKAM (Medicine), Carolyn PL Kng', \\ FRCP (Lond, Edin), FHKAM (Medicine), Jenny SW Lee ${ }^{5}$, MD, FRCP (London), \\ James KH Luk ${ }^{2}$, FRCP (Edin, Glasg, Ire), FHKAM (Medicine), Winnie YW Mok², \\ FRCP, FHKAM (Medicine), CK Shum ${ }^{6}$, MBBS, FHKAM (Medicine), CW Wong ${ }^{7}$, \\ FHKCP, FHKAM (Medicine) on behalf of The Hong Kong Geriatrics Society
}

\footnotetext{
Department of Medicine and Geriatrics, Pok Oi Hospital, Hong Kong

2 Department of Medicine and Geriatrics, Fung Yiu King Hospital, Hong Kong

3 Department of Medicine and Geriatrics, Kwong Wah Hospital, Hong Kong

4 Department of Medicine and Geriatrics, Ruttonjee \& Tang Shiu Kin Hospitals, Hong Kong

5 Department of Medicine, Alice Ho Nethersole Hospital, Hong Kong

6 Department of Medicine and Geriatrics, Tuen Mun Hospital, Hong Kong

7 Department of Medicine and Geriatrics, Caritas Medical Centre, Hong Kong
}

Correspondence to: Dr Tung Wai Auyeung, Department of Medicine and Geriatrics, Pok Oi Hospital, Hong Kong. Email: auyeungtw@ha.org.hk

Key words: COVID-19; Frail elderly; Infection control

\section{INTRODUCTION}

The first case of coronavirus disease 2019 (COVID-19) in Hong Kong was reported on 23 January 2020. On 25 January 2020, the government activated the emergency response level to combat COVID-19. The mortality rate of the COVID-19 was expected to be considerably higher in the older population, and the case-fatality rate was much higher especially in those aged $>80$ years $(15-20 \%)^{2}$ and those with comorbidities. ${ }^{3}$ In Hong Kong, as of 17 July 2020, there were 10 deaths out of 1655 confirmed cases. Seven of the 10 mortalities involved patients aged $\geq 70$ years. Social distancing as a strategy to prevent COVID-19 spread has affected the health and community services for older people. In 2004, The Hong Kong Geriatrics Society published a position statement on old patients with severe acute respiratory syndrome
(SARS). ${ }^{4}$ The guidelines were followed in face of the current COVID-19 pandemic. In the present paper, we report our experience of caring for older people in Hong Kong during the COVID-19 pandemic. We discuss the effects on older people of restricted access to health and community services and infection control measures in residential care homes for the elderly (RCHEs). We also review the treatment of older patients after admission to hospitals, their endof-life (EOL) care, and the emergence of telecare as a new service model.

\section{RESTRICTED ACCESSIBILITY TO HEALTH AND COMMUNITY SERVICES}

In response to the activation of emergency response level, the Hong Kong Hospital Authority suspended non-emergency and non-essential services to 
streamline staff and resources to respond to COVID-19.5 Geriatric day-hospital services were suspended. The Social Welfare Department also suspended community services for older people, including day-care centre services and outreach rehabilitation services run by non-governmental organisations. Integrated and enhanced home care services were limited to delivering meals, escort, nursing, and drug administration services. ${ }^{6}$

This restriction in access to health and social services have exerted physical, psychological, and social pressure on older people and their informal caregivers. For older people, these changes can result in exacerbation of chronic diseases (owing to less frequent monitoring or possible disruption in medication access), increased physical inactivity leading to frailty and sarcopenia (owing to suspension of geriatric day-hospital and outreach rehabilitation services), and reduced psychosocial support (worsening behavioural and psychological symptoms of dementia). For informal caregivers, because of sudden discontinuation of community services, they are obliged to provide more care and even change the types of care (eg, living with older people as a contingency plan to provide daily activities assistance). Family caregivers may be under dual stress from financial pressures and fear of infection in themselves (as no contingency plan if they are sick or being quarantined) and in the older family member (as they are vulnerable). They may also have to balance providing care and working from home at the same time, while also experiencing financial pressures due to decreasing or complete loss of income and increasing payments for food and personal protective equipment.

According to an online survey of caregivers' experience during COVID-19, 70\% of informal caregivers were providing more care than before, $35 \%$ of whom were providing more care as a result of local services reducing or closing. They were providing an average of 10 additional hours of care a week, and $55 \%$ of them felt overwhelmed and were going to burn out. ${ }^{7}$

The limited availability of community services has also affected discharge from hospitals. Older patients are at risk of functional decline after hospitalisation. ${ }^{8}$ With the deterioration of functionality to cope with activities of daily living, care needs are provided upon discharge. Integrated care and discharge support that coordinates community services and community nursing service can provide post-discharge care and support for those living in the community. ${ }^{9}$ These community services include home care services and day care centre services. In addition, geriatric day hospitals provide comprehensive geriatric assessment and rehabilitation for medically stable older patients. These support services enable timely hospital discharge with safer and better postdischarge care to prevent disease recurrence and readmission. However, the suspension of community services and other post-discharge continuous care options have affected the discharge planning of older patients from hospitals.

Although the integrated care and discharge support and community nursing service run by Hospital Authority have continued, without the support from the community services run by nongovernmental organisations, it is insufficient to meet the care needs of older patients on discharge, especially those living alone or only having minimal family support. The deficient or decreased support can be stressful and disruptive as family caregivers may not be able to afford longer time to take care of their older relatives.

For those planning to have post-discharge care in RCHE, the 'no visitor' policy ${ }^{10}$ prevents families from inspecting the environment, care standard, and cost of RCHE. The policy may also cause families to worry that patients will feel isolated or that the quality of care in the RCHE will be affected. Even if the family decides to hire a domestic helper to care for the patient, international travel and quarantine restrictions are affecting travel to and from Hong Kong. Therefore, families are reliant on hospitals to care for their older family members and this has led to delays in discharge.

\section{INFECTION CONTROL AT RESIDENTIAL CARE HOMES FOR THE ELDERLY AND COMMUNITY GERIATRICS ASSESSMENT TEAM SERVICES}

The high fatality rate among residents in long-term care facilities in United States and Europe has aroused much media coverage worldwide. Right from the early phase of this epidemic, the community 
geriatrics assessment teams (CGAT) in Hong Kong have been in high alert to prevent any outbreak in RCHEs. CGAT is an out-reach healthcare team, consisting of geriatricians, geriatric nurses, and allied health professionals, delivering on-site services to older persons living in RCHEs or old age homes. Older people living in RCHEs are at high risk of mortality and morbidity, owing to the poor health and functional status of the older residents and the crowded environment. During the pandemic of COVID-19, there is an imminent need to expand infection control measures and modify the workflow of CGAT in RCHEs accordingly.

The regular reporting mechanism of influenzalike illness incidents in RCHEs, which has been in place since 2003 SARS period, was reinforced, and any clustering of influenza-like illness would be recognised promptly and investigated jointly by CGAT and Centre for Health Protection. New measures have been used for infection control in RCHEs. CGAT has monitored RCHEs to comply with the guidelines for prevention of COVID-19. ${ }^{10}$ These include checking of body temperature of residents and staff every day; no visiting by outsiders; wearing of personal protective equipment when performing healthcare or personal caring procedures; advising residents to wear surgical masks where feasible; avoiding arranging non-essential group activity; installing partitions on dining tables to segregate diners; staggering meal times to avoid gathering in dining area; avoiding rotation and unnecessary deployment of staff; arranging for similar groups of staff to perform their duties in same area/floor to avoid cross contamination; advising staff to maintain social distancing and avoid chatting during meal times; immediately isolating residents with symptoms; promoting proper cleansing techniques in maintaining environmental hygiene; promoting proper hand hygiene and personal hygiene; maintaining good ventilation; handling residents newly discharged from hospital; and handling staff or residents returning from overseas. Wearing of personal protective equipment for CGAT staff was enforced to minimise the risk of cross-infection. On-site or telecare support was provided to manage any non-influenza-like illness to reduce accident and emergency department attendance and hospital admission. Some CGATs have segregated a group of designated geriatricians to deliver CGAT service from other in-patient duties to prevent cross-infection between hospital and RCHEs. All these infection control measures can limit the extent of contact tracing and quarantine if a case of COVID-19 occurs.

Although the government has put quarantine camps in place for close contact persons of COVID-19, they are unsuitable to house older people and in particular RCHE residents who need assistance in activities of daily living. On-site quarantine is also unfeasible, given the over-crowded conditions at most RCHEs and the limited human resources; care workers at RCHEs would have to be segregated in quarantine camps. A contingency plan is in place for RCHE residents confirmed to have COVID-19. On day 1, the local CGAT visits the RCHE together with Centre for Health Protection Infection Control Branch to assess the feasibility of on-site quarantine. A site for segregation has been identified and prepared to accommodate a certain proportion of the residents to decongest that RCHE. Whether placing the close contact or non-close contact residents on-site or in an off-site facility depends on the number of the respective proportion. The Social Welfare Department has contracted a team of care workers and nurses to manage the quarantine site. The local CGAT, of which the quarantine site is located, will provide regular medical and nursing services, drug refill service and advice, and facilitate hospitalisation if any residents have health deterioration.

In the first and second waves of the pandemic, Hong Kong had a low infectivity rate in RCHEs. Hong Kong learned a great deal during the 2003 SARS epidemic, when there were widespread outbreaks and mortalities in such long-term care facilities. In response to the current pandemic, RCHE operators implemented the 'no visitor' policy promptly and CGAT teams quickly implemented improved infection control measures. However, in the third wave, the first case of COVID-19 in an RCHE was diagnosed on 6 July 2020, nearly half a year after the first case occurred in Hong Kong. The third wave was related to the relaxing of social distancing and allowing visiting in RCHEs. At the time of writing this paper, 24 residents and 5 healthcare workers of this RCHE have been diagnosed with COVID-19.

\section{OLDER PATIENTS AFTER ADMISSION DURING THE COVID-19 PANDEMIC}

The COVID-19 pandemic has challenged elder- 
friendly care delivery. The Hospital Authority has enforced a strict 'no visitor' policy at hospitals. Routines of bedside family engagement for geriatric care, rehabilitation, EOL care, and discharge planning have been suspended. Infection control is the highest priority, superseding other practices and prioritised as a the 'new normal'. However, families have positive contributions to elderly care during hospitalisation. They participate in the therapeutic process, not only in physical provision of daily care such as feeding, oral hygiene, and cleansing but also in psychological and emotional support. They reduce patient stress and provide encouragement during recovery from illness. For patients with dementia, families provide collateral information about the illness and the person, and their familiar presence reduces the occurrence of delirium. Partnering with families is a positive enabler to quality and safe care. Loss of connection owing to the'no visitor' policy has severed these benefits and potentiated low mood, exacerbating a sense of abandonment, helplessness, and depression, with undesirable consequences on recovery, discharge, and EOL care processes.

Although a simple telephone call could connect older patients with their family, videoconferencing provides a far superior experience. However, many older people do not own smartphones or are unable to independently operate such devices or software for videoconferencing. Thus, telemedicine may heighten inequities in elderly care where such access gaps are widened further. Technology alone cannot help; rather, staff experienced in elderly care are needed to embed such important connections into the elderly care process in the era of COVID-19.

During the COVID-19 emergency response level activation, any patients with respiratory symptoms or fever are admitted to surveillance wards where infection control takes priority over privacy, comfort, and dignity. Older patients are one of the most frequently seen patient groups in the surveillance and isolation wards although most confirmed cases have been otherwise healthy younger people who have returned from abroad or who have had social contact with returnees. The treatment of these older patients has been very different from the usual practice in acute geriatric wards. Normally doctors would look for any cause of sepsis or dyspnoea, but in isolation or surveillance wards, the focus is on taking nasopharyngeal and throat swabs and obtaining results quickly. These older patients are treated in the same way as younger patients, except that they have difficulty responding to doctors and nurses via the intercom or communicating with their families via the internet. Monitoring, blood checks, and medical treatment are administered aggressively. Decisions are made with a focus on patient disposal: was the patient 'clean'? Could the patient be 'stepped-down' to the general ward to make the bed available for another potential case?

Different uncomfortable and intrusive procedures including nasopharyngeal swabs, blood tests, or even computed tomographic scanning of the thorax might be carried out to rule out COVID-19 infection, irrespective of their underlying illnesses and pre-morbid status. They might be transferred to other wards once COVID-19 was excluded, usually within a few hours. The frequent and rapid transitions of older frail patients in hospitals might lead to delirium, particularly in those with advanced dementia, and hospital complications.

If the COVID-19 test result was not yet available and the patient was dying, family would not be allowed to visit the patient even at this last moment. It was only when the test was negative, then the family would be allowed to see the dead body and say goodbye to their beloved older family member.

Below is a narrative from a senior geriatrician describing her experience on a surveillance ward caring for older patients:

"I was disorientated on the first day, engrossed in an atmosphere of efficient and protocol-driven medicine which was very new to me. A terminal lung cancer patient was admitted to our isolation ward due to dyspnoea with a bloody pleural effusion., On day two, he reminded me of my role as a geriatrician, though he was only 56. I discovered that the staff there knew very little about end-of-life care. I spoke to the patient about his grave prognosis and imminent death, explored his last wishes, withdrew futile monitoring and treatment, and allowed his wife to stay with him in his last hours. Over the next two weeks I continued to see patients who were critically ill, requiring noninvasive ventilatory support or intubation, but who were declined admission to the intensive care unit due to frailty or advanced diseases. Many of them were over 80 . Some of them were from old age homes. In my 
brief experience in these isolation wards, I could truly grasp the meaning of "isolation". In these purposebuilt rooms, human contact is a rare entity. Separated by glass panes and hooked up to monitors, patients with communication barriers, be it technological or verbal, received an efficient yet dehumanising medical processing. When the world is focusing on the safety of the masses, the frail, the silent and the old have no voice. When the next epidemic comes, I do hope that we could have some elderly-friendly isolation wards. After all, most elderly die of infections, and we have been very lucky that this time, as of today, the infection has not struck our nursing homes."

\section{END-OF-LIFE CARE IN THE COMMUNITY AND IN HOSPITALS}

The COVID-19 emergency response level has affected EOL care for older patients in the community and in hospitals. The CGAT support for EOL patients in RCHEs has been implemented by HA since 2015. ${ }^{11}$ The current'no visit' policy in most RCHEs has made the discussion of advance care planning and 'do not attempt cardiopulmonary resuscitation' with family members by CGAT doctors and nurses in RCHEs impossible. With the'no visitor' policy in place, nurses or medical social workers rely on telecommunication using tablet computers or mobile phones to allow patients to communicate with their families. However, this technology is not a replacement for face-to-face meetings with family members. Feeding problems including dysphagia or poor oral intake are common in older patients with advanced dementia or when they have reached the EOL stage. In recent years, careful hand feeding has become more favourable than nasogastric tubes for feeding. ${ }^{12}$ Family members play an important role in carrying out careful hand feeding to older patients. Because of the 'no visit' policies, carers and family members are unable to visit or feed the older patients. All careful hand feeding must be done by nursing staff, denying the last touching and caring experience of the family members to their beloved older but dying relatives. Family members might be allowed to see their dying relatives only at the last moment on compassionate grounds. When relatives arrive, the patient might have already become too weak to speak or may even be unconscious, depriving them of a final chance to say goodbye to family members. Spiritual and volunteer services have nearly come to a halt during the COVID-19 emergency response period. Spiritual and emotional support is unavailable to patients on their final journey of life in hospitals. Such support is allowed only when the patients are expected to die within hours, and with emergent permission by hospital chief executives.

\section{EMERGENCE OF TELECARE}

Some RCHEs have declined outreach CGAT visits for fear of cross infection with COVID-19. Teleconsultation via videoconferencing has emerged as a highly desirable alternative to provide medical care to RCHE residents. This mode of consultation was underused in the past due to costly hardware, unreliable connections, and security concerns during data transmission. During the current COVID-19 pandemic, mobile devices, high speed internet connections, and video communication software are readily available, so the number of tele-consultations has increased greatly in some hospitals.

Residents and staff of RCHEs have reported being very satisfied with the increase in teleconsultations. Mobile devices have been used by CGAT nurses to capture images of wounds for real-time consultations by wound specialists in hospitals. Telemedicine has also been used in virtual fast track clinics in integrated care and discharge support programmes, which include home visits by case managers, rehabilitation, and home care support by non-governmental organisations. Concurrent medical consultations via videoconferencing have been conducted during home visits by case managers. Patients with deteriorating health conditions can be assessed and prescribed treatment by doctors without the need to travel to hospitals. Moreover, in some integrated care and discharge support teams, regular multidisciplinary conferences with home support teams have been converted to video conferences, and these have been found feasible, efficient, and effective.

Before expanding this model of care in the 'new normal' phase, the associated challenges should be recognised. Firstly, older patients with comorbidities may be unable to operate devices independently due to cognitive, functional, or sensory impairment. Secondly, a structure and framework should be established to better define target patients, logistics, patient's acceptance, efficacy as well as medico-legal issues. Lastly, treatment outcomes should be 
evaluated to ensure that the level of care in telemedicine is comparable to on-site consultation. Ethical guidelines on the practice of telemedicine were published by the Medical Council of Hong Kong in 2019. ${ }^{13}$ The importance of the doctor-patient relationship, informed consent, privacy, security of records, and treatment evaluation were highlighted. Telemedicine cannot substitute the majority of direct patient care to older people. However, with increasing demand of healthcare services from the aging population, this COVID-19 pandemic has provided a catalyst for us to develop a complementary model to improve the efficiency, safety, and quality of care provided to older patients.

\section{CONCLUSION}

Many hospital practices and procedures, which were already unfriendly for older patients, have deteriorated owing to the effects of the current COVID-19 pandemic, whereby infection control takes priority over humanistic considerations. However, the emergence of telecare offers an opportunity for the development of a new service model to provide care for the older population.

\section{REFERENCES}

1. CE announces activation of Emergency Response Level in relation to novel coronavirus infection. Available from: https:// www.info.gov.hk/gia/general/202001/26/P2020012600087.htm. Accessed 20 July 2020.

2. Onder G, Rezza G, Brusaferro S. Case-fatality rate and characteristics of patients dying in relation to COVID-19 in Italy. JAMA 2020;323:1775-6. Crossref

3. Jordan RE, Adab P, Cheng KK. Covid-19: risk factors for severe disease and death. BMJ 2020;368:m1198. Crossref

4. The Hong Kong Geriatrics Society. Position statement on severe acute respiratory syndrome (SARS) in elders. J Hong Kong Geriatr Soc 2004;12:37-42.

5 Hospital Authority adjusts service provision to focus on combatting epidemic. Available from: https:/www.info.gov.hk/ gia/general/202002/10/P2020021000711.htm. Accessed 20 July 2020.

6. Special arrangements for subvented welfare services and services of Social Welfare Department after Chinese New Year holidays. Available from: https://www.info.gov.hk/gia/general/202001/28/ P2020012800751.htm. Accessed 20 July 2020.

7. Caring Behind Closed Doors. Available from: https://www. carersuk.org/images/News_and_campaigns/Behind_Closed_ Doors_2020/Caring_behind_closed_doors_April20_pages_web_ final.pdf. Accessed 20 July 2020.

8. De Saint-Hubert M, Schoevaerdts D, Poulain G, Cornette P, Swine C. Risk factors predicting later functional decline in older hospitalized patients. Acta Clinica Belg 2009;64:187-94. Crossref

9. Lin OY, Luk KH, Chan TC, Mok WY, Chan HW. Effectiveness of a discharge planning and community support programme in preventing readmission of high-risk older patients. Hong Kong Med J 2015;21:208-16. Crossref

10. Centre for Health Protection, Department of Health, Hong Kong SAR Government. Guidelines for residential care homes for the elderly or persons with disabilities for the prevention of coronavirus disease. Available from: https://www.chp.gov.hk/ files/pdf/advice_to_rche_rchd_on_prevention_of_nid_Jeng.pdf. Accessed 20 July 2020.

11. Luk JKH, Chan FHW. End-of-life care for advanced dementia patients in residential care home - a Hong Kong perspective. Ann Palliat Med 2018;7:359-64. Crossref

12. Luk JKH, Chan FHW, Hui E, Tse CY. The feeding paradox in advanced dementia - a local perspective. Hong Kong Med J 2017;23:306-10. Crossref

13. The Medical Council of Hong Kong. Ethical Guidelines on Practice of Telemedicine. Available from: https://www.mchk.org. hk/files/newsletter-26th.pdf. Accessed 20 July 2020. 